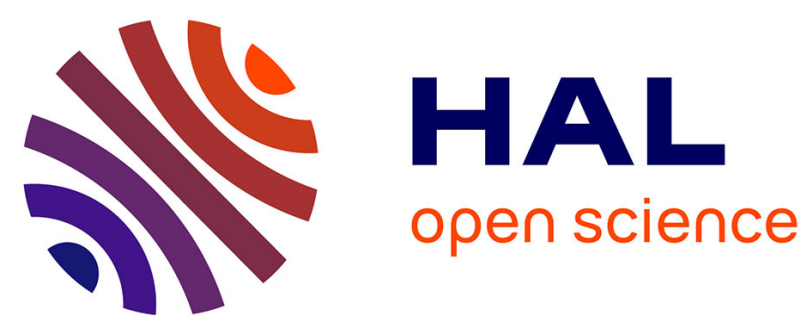

\title{
Tailoring the Composition of Eu(3+)-Doped Y3NbO7 Niobate: Structural Features and Luminescent Properties Induced by Spark Plasma Sintering.
}

Ka-Young Kim, U-Chan Chung, Brice Mutulet, François Weill, Alain

Demourgues, Julie Rossit, Jean-Marc Heintz, Amélie Veillere, Veronique

Jubera

\section{To cite this version:}

Ka-Young Kim, U-Chan Chung, Brice Mutulet, François Weill, Alain Demourgues, et al.. Tailoring the Composition of $\mathrm{Eu}(3+)$-Doped Y3NbO7 Niobate: Structural Features and Luminescent Properties Induced by Spark Plasma Sintering.. Inorganic Chemistry, 2017, 56 (8), pp.4495-4504. 10.1021/acs.inorgchem.7b00088 . hal-01520638

\section{HAL Id: hal-01520638 \\ https://hal.science/hal-01520638}

Submitted on 11 May 2017

HAL is a multi-disciplinary open access archive for the deposit and dissemination of scientific research documents, whether they are published or not. The documents may come from teaching and research institutions in France or abroad, or from public or private research centers.
L'archive ouverte pluridisciplinaire HAL, est destinée au dépôt et à la diffusion de documents scientifiques de niveau recherche, publiés ou non, émanant des établissements d'enseignement et de recherche français ou étrangers, des laboratoires publics ou privés. 


\section{Tailoring the composition of $\mathrm{Eu}^{3+}$-doped $\mathrm{Y}_{3} \mathrm{NbO}_{7}$ niobate: structural features and luminescent properties induced by spark plasma sintering}

Ka-Young Kim ${ }^{\dagger \ddagger}$, U-Chan Chung ${ }^{\dagger \ddagger}$, Brice Mutulet ${ }^{\dagger \ddagger}$, François Weill ${ }^{\dagger \ddagger}$, Alain Demourgues ${ }^{\dagger \ddagger}$, Julie Rossit ${ }^{\dagger \ddagger}$, Jean-Marc Heintz $^{\dagger \ddagger \S}$, Amélie Veillere ${ }^{\dagger \ddagger \S}$, and Véronique Jubera ${ }^{\dagger \ddagger}$

${ }^{+}$CNRS, ‡Univ. Bordeaux, and §Bordeaux INP, ICMCB, UPR9048, F-33600 Pessac, France

\section{Abstract :}

The defective fluorite-related $\mathrm{Y}_{3} \mathrm{NbO}_{7}$ host lattice was doped with $\mathrm{Eu}^{3+}$ ions to understand the influence of spark plasma sintering (SPS) process on this host lattice. The intrinsic disorder due to the occurrence of oxygen vacancies results in amorphous-type responses of the luminescent cations, and the spectral distribution varies as a function of the niobium content. Two spectral fingerprints of europium emissions were clearly enhanced. The correlation between luminescence, $\mathrm{X}$-ray diffraction, and electron diffraction characterizations shows the existence of local inhomogeneity. Indeed, the particular nonequilibrium sintering conditions allowed pointing out a lack of miscibility within the $\mathrm{Y}_{3} \mathrm{NbO}_{7}$ solid solution domain. Thus, the SPS pellet is a composite of two extreme compositions. This phase demixing is mainly induced by the pressure coupled with a current effect that makes possible ionic migration in this $\mathrm{Y}_{3} \mathrm{NbO}_{7}$ ionic conductive matrix.

\section{Introduction}

The $\mathrm{Re}_{3} \mathrm{NbO}_{7}$ (Re, rare earth) niobate family presents peculiar crystallography features, as structural studies showed. For the largest rare earth matrix (from La to $\mathrm{Nd}$ ) the compounds crystallize in the orthorhombic Pnma space group. It adopts the C2221 space group for $\mathrm{Re}=\mathrm{Sm}-\mathrm{Tb}$ and finally a cubic $\mathrm{Fm} 3 \mathrm{~m}$ symmetry for the smallest Re $=Y$, Dy-Lu. $(1,2)$ In the case of the cubic symmetry $(\operatorname{Re}=Y, L u, Y)$, electron diffraction and high-resolution transmission electron microscopy (HRTEM) characterization revealed local nanodomains that were related to the pyrochlore network (space group $\mathrm{Fd} 3 \mathrm{~m}),(3,4)$ even if some authors announced that the too-low intensity of the additional diffraction spots are not consistent with the reflections of the $F d 3 m$ space group. $(5,6)$ Complementary Raman spectroscopy confirms the position of vibrational modes, which excludes a pure fluorite structure.(7)

Because trivalent europium luminescence is sensitive to the local environment, yttrium matrix has been doped with this element to investigate this structure.
We recently published first results of the europium emission as a function of the temperature.(8) This study reports a maximum of intensity due to the ${ }^{5} D_{0}-$ ${ }^{7} \mathrm{~F}_{2}$ electric dipole transitions. The global spectral distribution shows a thermal evolution of the solidstate solution, and a decomposition is detected for the longest thermal treatment above $1100{ }^{\circ} \mathrm{C}$. This results in the appearance of emission peaks associated with the location of $\mathrm{Eu}^{3+}$ ions in $\mathrm{Y}_{2} \mathrm{O}_{3}$ and $\mathrm{YNbO}_{4}$ phases resulting from the demixing phase process.

Powder material was sintered through spark plasma sintering (SPS) process to ensure a high crystallization and favor the stabilization of the rare earth environment within the host. A pellet that does not present trace of impurity regarding the optical response was selected for the present investigation. We propose here a correlation between the optical response, the structural characterization performed through scanning electron microscopy (SEM), X-ray diffraction, TEM, and the SPS process. 


\section{Synthesis}

The experimental procedure to stabilize the $\mathrm{Eu}^{3+}$ doped $\mathrm{Y}_{3} \mathrm{NbO}_{7}$ powder is described elsewhere.(8) The purity of the starting materials was $99.99 \%$ for the oxides $\mathrm{RE}_{2} \mathrm{O}_{3} \mathrm{RE}=$ lanthanides, $99.998 \%$ for $\mathrm{NdCl}_{5}$, 99\% for ethylene glycol, $99.5 \%$ for citric acid, and 99.9\% for methanol.

As the stability of the niobate is announced for $\mathrm{Nb} /[\mathrm{Nb}+\mathrm{Y}]$ atomic ratio between 0.21 and 0.28 at $1700{ }^{\circ} \mathrm{C},(9,10)$ we fixed the final cation proportion at 0.25 to obtain the $\mathrm{Y}_{2.85} \mathrm{Eu}_{0.15} \mathrm{NbO}_{7}$ composition.

SPS experiments were performed in a Syntex $\mathrm{Dr}$ Sinter $515 S$ machine. Powders were heated at 5 ${ }^{\circ} \mathrm{C} / \mathrm{min}$ to $1600{ }^{\circ} \mathrm{C}$ and held 20 min under primary vacuum. A $100 \mathrm{MPa}$ pressure was applied uniaxially from room temperature during all the sintering process. After the sintering, the ceramics were annealed at $900{ }^{\circ} \mathrm{C}$ under oxygen during $2 \mathrm{~h}$ to remove carbon contamination. Then, the ceramics were mirror polished on both sides using diamond slurry.

\section{SEM Pictures}

The powder morphology and the microstructure of the sintered pellets were observed using SEM (TESCAN Vega II SBH SEM). Powders and ceramics were previously coated with gold prior to their characterization. Sintered samples were observed either on the surface after polishing and thermal revelation ( $30 \mathrm{~min}$ at $T_{\text {sintering }}-50{ }^{\circ} \mathrm{C}$ ) or directly on a fractured sample.

\section{Results and Discussion}

\section{SEM Pictures}

The initial europium-doped niobate powder, prepared by a Sol-Gel type of method, is presented in amount of intergranular porosity with a spherical shape can be observed, which is consistent with the

The SPS sintering process induced an increase of the grain size and a large distribution in sizes, ranging from $400 \mathrm{~nm}$ to $2 \mu \mathrm{m}$ (Figure $1 \mathrm{c}, \mathrm{d}$ ).
The photoluminescence properties were analyzed using a SPEX FL212 spectro-fluorimeter. The excitation spectra were corrected for the variation of the incident flux; the emission spectra were corrected for the transmission of the monochromator and the response of the photomultiplier. The equipment used consisted of a $450 \mathrm{~W}$ xenon lamp, an excitation double monochromator, an emission double monochromator, and a thermoelectrically cooled photomultiplier tube. Low-temperature measurements were collected with an Oxford cryostat connected to the previous equipment.

\section{X-ray Diffraction Analysis}

Room-temperature powder X-ray diffraction (XRD) patterns were collected on a PANalytical X'pert PRO MPD using a Bragg-Brentano $\theta-2 \theta$ geometry and equipped with a $\mathrm{Ge}(111)$ monochromator to obtain a monochromatic $\mathrm{Cu} \mathrm{Ka1}(\lambda=1.54059 \AA)$. The europium/yttrium niobate matrix diffractogram was refined using the Rietveld method with the conventional reliability factors. The Fullprof program package was used.(11) The unit cell parameters, atomic positions, and Debye-Waller factors were refined on the basis of the $\mathrm{Fm} 3 \mathrm{~m}$ space group corresponding to the fluorite-type structure.

\section{Electron Diffraction Analysis}

Electron diffraction patterns were collected on a JEOL 2100 transmission electron microscope, working at $200 \mathrm{kV}$ and equipped with a double tilt specimen stage. Prior to its observation, a suspension of the specimen in alcohol was prepared, and a drop was deposited on a carbon-coated grid.

Figure 1a,b. These micrographs show micrometric agglomerates, made of nanometric grains with homogeneous size between 80 and $100 \mathrm{~nm}$. Figure $1 c, d$ shows the surface of the dense ceramics obtained after SPS sintering process. A low

high relative density (98\%) measured by the Archimedes method.

The fracture of the sintered material is occurring through an intergranular mode, as it can be seen in Figure 1e. It is a classic feature for dense ceramic materials, and it is consistent with the high density 
obtained in our case. These micrographs also reveal a good densification in the volume of the samples.

\section{Luminescence Characterization}

Excitation and emission spectra obtained at room temperature on the $\mathrm{Eu}^{3+}(5 \mathrm{~mol} \%)$-doped $\mathrm{Y}_{3} \mathrm{NbO}_{7}$ pellet are shown in Figure 2.

Excitation performed at $290 \mathrm{~nm}$ revealed a maximum of the emission due to the hypersensitive ${ }^{5} D_{0}-{ }^{7} F_{2}$ electric dipole transition. As observed on powder in a previous work, the spectral distribution is quite large and similar to that observed in an amorphous or lowcrystallized environment.(8) No clear discrimination of the Stark component splitting can be done under such an excitation wavelength. The excitation spectrum is constituted by large UV absorption bands ranging between 230 and $300 \mathrm{~nm}$. The absorptions are linked to the electron charge transfer from the oxygen ligand (charge transfer band, CTB) to the empty orbital of europium ions at the highest energies(12) and probably to the charge transfer from oxygen to niobium at the lowest energies.(13) In addition to the large band, $4 \mathrm{f}-4 \mathrm{f}$ absorption lines of the rare earth ions are detected. Their intensities are enhanced compared to that of the UV CTB due to a configurational quenching of the emission, which results in a direct nonradiative de-excitation from the CTB to the ${ }^{7} F_{J}$ fundamental states. This nonradiative process is by consequence a drawback that forbids the use of such red-emitting phosphor for application requiring high quantum efficiency.

Selective excitations were performed to obtain a more precise resolution of the emission graph. Two spectral distributions were isolated under excitation at $462 \mathrm{~nm}$ and at $468 \mathrm{~nm}$. The corresponding emission graphs are reported in Figure $2 b$. The two $\mathrm{Eu}^{3+}$ fingerprints revealed that the anionic polyhedra around the doping element possess two specific geometries that induce changes of the Stark component splitting. None of these sites seem to be well-defined, as the width of each peak is large, and the intensity of the electric dipole transitions reveals a lack of inversion center. These measurements were repeated at liquid He temperature $(4 \mathrm{~K})$ to reduce the thermal contribution to the global emission broadening.

Thermal evolution of surface and bulk response is reported by Montini et al.(17) Among all the pyrochlore structures, the $\mathrm{La}_{4} \mathrm{Gd}_{11} \mathrm{EuHf}_{16} \mathrm{O}_{56}$ Hafnate or the $\mathrm{La}_{4} \mathrm{Gd}_{11} \mathrm{EuZr}_{16} \mathrm{O}_{56}$ zirconate present an optical response quite similar to the $\mathrm{Eu}^{3+}$-doped $\mathrm{Y}_{3} \mathrm{NbO}_{7}$. But
Excitation graph, illustrated in (a-left), shows a higher contribution of the Eu-O CTB. Excitations in the CTB or in the $4 f$ lines are shown in (a-right) and (b, c) respectively. (d) illustrates an intrinsic emission of the niobate matrix. Figure 3a-c illustrates the excitation and emission graphs related to the europium luminescence, measured at $4 \mathrm{~K}$. One can observe on the excitation curve (Figure 3a) a clear increase of the CTB intensity in comparison to that of the $4 \mathrm{f}-4 \mathrm{f}$ absorption lines. This confirms the high impact of a too-low location of the CTB, which contributes to the strong configurational quenching observed at room temperature. The decrease in temperature does not reveal a better structuration of the lines. Selective excitation and emission spectra were performed to definitely attribute the nature of the observed lines. Corresponding graphs are reported in Figure S1.

At this concentration ( $5 \%$ of molar substitution equivalent to 1021 ions $\cdot \mathrm{cm}^{-3}$ ), radiative de-excitation from ${ }^{5} D_{2}$ and ${ }^{5} D_{1}$ level is still detected between 480 and $570 \mathrm{~nm}$ (Figure 3c) under excitation in the ${ }^{5} \mathrm{D}_{2}$ level.

Regarding the global emission, it is quite unexpected to obtain such a proportion of the electric dipole transition of $\mathrm{Eu}^{3+}$ within a fluorite matrix. Indeed, one can totally exclude the location of europium ions in the cubic or octahedral regular cationic environment of the fluorite host or with the sixfold and eightfold coordination polyhedra of the pyrochlore structure that often results from the deformation of the fluorite structure. In this latter case, the larger site possesses a $D_{3 d}$ symmetry. The ${ }^{5} D_{0}-{ }^{7} F_{1}$ magnetic dipole transitions split into $A$ and $E$ component. The energy difference between the two lines was studied in different pyrochlore matrices, and it was shown that it varies as a function of the radius size difference between the $A$ and $B$ cations of the $\mathrm{A}_{2} \mathrm{~B}_{2} \mathrm{O}_{7}$ compounds. $(14,15)$ The luminescence of europiumdoped pyrochlore is mainly due to the magnetic dipole transitions, and the corresponding emission is orange. Moreover, a few examples of red emission of fluorite or pyrochlore can be found in the literature. The local symmetry is lowered to the Td punctual symmetry for most distorted polyhedra in tetragonaltype $\mathrm{Ce}_{y} \mathrm{Zr}_{1-y} \mathrm{O}_{2}$ solid solution.(16) $\mathrm{Ce}_{y} \mathrm{Zr}_{1-y} \mathrm{O}_{2}$ presents a red surface emission due to disturbed local sites.

the authors point out a low crystalline rate of the studied materials and the occurrence of amorphoustype environments that can justify the lack of symmetry around the rare earth element.(14) These optical characterizations definitively exclude the 
location of $\mathrm{Eu}^{3+}$ ions in pyrochlore-type environments.

Considering the observed emission of $\mathrm{Eu}^{3+}$-doped niobate, we cannot exclude the fact that each of the observed 1 and 2 emissions is associated with a unique environment, as selective excitations show modification in the intensity ratio of the electric dipole transitions (Figure S2). But no fingerprint of the expected secondary phases as $\mathrm{Eu}^{3+}$-doped $\mathrm{Y}_{2} \mathrm{O}_{3}$ or $\mathrm{YNbO}_{4}$ is detected. At this stage and knowing that thermal treatment affects the spectral distribution as previously demonstrated, we performed complementary structural characterizations to identify the optical response of europium ions in this material.

In addition, the low temperature makes possible the observation of a large emission band related to the niobium-oxygen charge-transfer emission (Figure 3dright, blue curve). The corresponding absorption consists of a large band, peaking at $\sim 300 \mathrm{~nm}$ (Figure 3a-left, black curve). Such charge-transfer emission of anionic groups is well-known in molybdate or tungstate host lattice, and because of a large Stokes shift, it is generally strongly quenched at room temperature.(18-20) Recently it has been reported in the $\mathrm{YNbO}_{4}$ matrix.(13)

\section{X-ray Diffraction Characterization}

Synthesis of the pure $\mathrm{Eu}_{3} \mathrm{NbO}_{7}$ compound was performed through the same synthetic route as the yttrium phase to detect if the introduction of $5 \%$ of europium could reduce the cubic symmetry of the yttrium niobate phase. Indeed, the europium phase is announced to be orthorhombic and crystallizes with the C2221 space group.(2) Experimental diffraction peaks perfectly match with the cubic yttrium phase crystallizing in the $\mathrm{Fm} 3 \mathrm{~m}$ space group (Figure S3). As a consequence, it can be said that the $\mathrm{Y}^{3+}$ substitution by $\mathrm{Eu}^{3+}$ ions will not affect the global symmetry of the host.

Full pattern matching and Rietveld refinements were then performed on a crushed pellet sintered in the same SPS conditions as the one studied before. A $1 \mathrm{~h}$ longer postheat-treatment under oxygen at $900{ }^{\circ} \mathrm{C}$ was performed on this sample in comparison with the one described in Figures 1 to 3 . The objective of the postheat-treatment was to reoxidize the pellet after 5.2525(9) and 5.2398(2) $\AA$, which correspond to $\mathrm{Nb} /[\mathrm{Nb}+\mathrm{Y}]$ ratios equal to 0.246 and 0.286 , respectively, according to J.-H. Lee et al. Because the proportion of fluorite 1 represents $77.9 \%$ of the total the reducing SPS sintering process. Results are illustrated in Figure 4.

The longer postheat treatment results in a light decomposition of the niobate phase, and traces of the fergusonite are visible on the Figure $4 b$ on both sides of the main diffraction peak of the niobate phase. Moreover, the monochromatic-type acquisition makes possible the observation of diffraction peaks splitting, at the highest angles range, which reveals the stabilization of two distinct fluorite-type compounds. By consequence, the refinements were run with two fluorite unit cells crystallizing in the $\mathrm{Fm} 3 \mathrm{~m}$ space group. The data corresponding to the full pattern matching and Rietveld refinements are reported in Table 2.At this stage, different XRD patterns were run on several powders, containing different niobium content and heat-treated at $1600{ }^{\circ} \mathrm{C}$, to show the variation of the cell parameters as well as the shape of diffraction peaks at high angles as a function of the niobium content. Under an equivalent heat-treatment performed in a classical furnace during the same time, no splitting of the diffraction peaks can be distinguished on the X-ray diffraction patterns (Figure S4). But for the lowest niobium content (21\%), traces of $\mathrm{Y}_{2} \mathrm{O}_{3}$ are detected, whereas the X-ray pattern reveals the predominance of fergusonite impurities in the highest niobium content (27\%) compound. Barker et al. and J.-H. Lee et al. showed that the $a$ parameter of the fluorite cubic solution decreases from 5.264 to $5.243 \AA$ in the 20 to $28 \%$ of niobium molar percentage range. $(10,21)$ Figure 5 reports our experimental results in the $21 \%-27 \%$ targeted range of niobium ratio. Unfortunatly our samples, with 21 and $27 \% \mathrm{Nb}$ ratio, underwent a decomposition giving rise to $\mathrm{Y}_{2} \mathrm{O}_{3}$ and/or $\mathrm{YNbO}_{4}$ under the $1600{ }^{\circ} \mathrm{C}$ heattreatment; the corresponding refined cell parameters converge to a value that is consistent with $24 \%$ of niobium content. However, a $23 \%$ niobium content compound was obtained on a material treated at lower temperature $\left(900^{\circ} \mathrm{C}\right)$ and for which no trace of impurity was detected. This last point matches perfectly with the linear law obtained by Barker and Lee.The cell parameters of the two fluorite-type host lattices stabilized in the pellet after SPS cycling are distributed on both sides of the linear law, which confirms the evolution of the composition during the sintering process. Lebail cell parameters refinement converge to cell parameters equal to

fluorite amount (Rietveld refinement), the balanced calculated value obtained is equal to $5.25 \AA$, which is close to the expected value. The highest proportion of poor niobium content fluorite is in agreement with 
the detection of the fergusonite phase detected by $X$ ray diffraction in this pellet.

This fluorite-type host lattice is known to present ionic conductivity properties. Because of oxygen mobility within the solid solution, the anionic conductivity was demonstrated by J.-H. Lee et al.(10) for several niobium contents. As a consequence, we suggest that the SPS sintering process, which implies current circulation from the top side to the back side of the pellet, forces oxygen mobility and cation diffusion, and favors the cubic fluorite-type solid solution splitting into two different compositions.

In addition, an enlargement in the low-intensity diffraction zone revealed additional large contribution, which cannot be taken into account by the two defined fluorite composition or cannot be explained by a modification of the symmetry like pyrochlore deformation ( $F d 3 m$ space group), fluorite defective matrix as sesquioxide (la3) or a lowering of the symmetry as for the $\mathrm{Gd}_{3} \mathrm{NbO}_{7}$ matrix $(\mathrm{Ccmm}$ space group; Figure S5). Therefore, complementary characterizations were performed by TEM to detect potential inhomogeneity at grain scale within the sintered pellet.

\section{Electron Diffraction}

TEM of several pyrochlore and fluorite cubic networks are reported in the literature. $(3,6,22)$ In most of them and especially in the $\mathrm{RE}_{2} \mathrm{O}_{3}-\mathrm{Nb}_{2} \mathrm{O}_{3}$ systems, additional diffraction spots attributed to superstructure microdomains existing in fixed orientation or superstructure are discussed. Concerning the $\mathrm{Y}_{3} \mathrm{NbO}_{7}$ matrix, Allpress et al. and Miida et al. described several weak and diffuse spots in addition to the strong spots, typical of the fluorite phase. $(4,5)$ These spots cannot match with those of an orthorhombic or pyrochlore structure.

Figure 6 illustrates electron diffraction patterns obtained on the SPS sintered crushed pellet. Nevertheless, differences exist from one grain to another one as illustrated in Figure 6a,b. These two patterns correspond to the same zone axis. Strong

High-resolution images would have been useful to get an idea of the extension of the local ordering. Unfortunately, probably because of SPS sintering, no crystals thin enough to obtain such images could be found on the TEM grid.

Such discrepancy concerning regular fluorite array was already mentioned by several authors who and sharp Bragg reflections are clearly consistent with the fluorite $\mathrm{Fm} 3 \mathrm{~m}$ cell. However, while only the $\mathrm{Fm} 3 \mathrm{~m}$ reflections are observed in Figure $6 \mathrm{a}$, diffused but localized spots are seen in Figure $6 \mathrm{~b}$.

The orientation of the latter crystal in the microscope led to patterns $6 \mathrm{c}, \mathrm{d}$. In these patterns, the more intense reflections belongs to the $\mathrm{Fm} 3 \mathrm{~m}$ cell. Additional double spots as well as rings are clearly visible. The double spots lie systematically around the position $\{1 / 21 / 21 / 2\}$. The distance between the two spots of the doublet is $\sim 0.45 \mathrm{~nm}^{-1}$, and this distance is always the same whatever the orientation of the pattern. In Figure $6 \mathrm{c}$ in addition to the double spots already described, one can observe a ring centered at position $\{1 / 23 / 21 / 2\}$. The diameter of the ring, $\sim 0.45 \mathrm{~nm}^{-1}$, is comparable to the distance between the two spots of a doublet. From these observations one can conclude, as Withers et al.(23) already did, that the additional doublets are part of a ring of diffuse intensity. From the fluorite structure, two types of superstructures have been identified: the socalled C-type (Ia3 Bixbite; $a=2 \mathrm{aF}$ ) structure and the $(F d 3 m ; a=2 \mathrm{aF})$ pyrochlore structure. The former is characterized by two sets of additional reflections localized at $1 / 2\{220\}$ and $\{001\}$. The latter gives also rise to two sets of superstructure spots but located at $1 / 2\{111\}$ and $\{001\}$. The observed diffuse rings appear around the expected positions for the ideal pyrochlore superstructure. However, we observed diffuse rings and not ideal spots, which indicates that there is only a local ordering. Also note that we did not observe any spot or diffusion at the $\{001\}$ position, which is consistent with the observation of Lopez-Conesa et al.(3)

At least, it should be noted that the very weak diffusion observed in the $\langle 220\rangle^{*}$ directions on the $6 \mathrm{~b}$ pattern is no more present on the $6 c$ pattern. Two explanations can be proposed: either it is a very localized phenomena and the two diffraction patterns are not obtained exactly from the same area or, as the orientation of the crystal is different, the projected thickness of the specimen is different and does not allow the observation of a so weak phenomena.

observed that Raman spectroscopy results were not consistent with the $\mathrm{Fm} 3 \mathrm{~m}$ space group. Because of their compatibility with the $F d 3 m$ symmetry, they were attributed to a pyrochlore structure and to a local ordering of the vacancies leading to domains the sizes of which are inferior to the sensitivity of the Xray analysis. $(7,24)$ 


\section{Discussion}

As demonstrated by the structural characterizations, the composition of the $\mathrm{Y}_{3} \mathrm{NbO}_{7}$ intermediate compound can be modulated by the SPS heat treatment, which favors oxygen mobility under current. The electronic diffraction microscopy reveals in addition to the typical satellite reflections of defect-type fluorite structure, inhomogeneity between grains belonging to the same pellet. As a consequence, the two spectral distributions of the europium emission could be attributed to the location of the europium ions into the two fluoritetype host lattices. The emission spectroscopy of trivalent europium reflects the dual nature of the sintered pellet, as two extreme spectral distributions are observed. It can be reasonably said that it also reflects the gradual transition between two different compositions, which results in a large and undefined spectral distribution of the $\mathrm{Eu}^{3+}$ radiative transition.

The luminescence of the $21 \%$ and $27 \%$ niobium content (targeted niobium content) was checked to determine if stabilization into two fluorite unit cells is linked to a thermal demixing in addition to the SPS effects (Figure 7). In both cases, as for the $25 \%$ niobium content powder studied in ref 8 , a unique large emission response is observed, whatever the excitation wavelength (Figure $7 c, d$ ). The spectral distributions indicate a small shift of the maximum between the two niobium content powders (Figure 7a). The emission 1 (see Table 1) is lightly closer to the highest niobium content, whereas the emission 2 better matches with the $21 \%$ of niobium content (Figure 7a). A systematic study as a function of the niobium content will be run to determine the attribution of emissions 1 and 2 regarding the niobium concentration. At this step, we can say that in the defective oxygen host, the occurrence of $\mathrm{NbO}_{6}$ octahedra stabilized into a fluorite-type network contributes to create distortion of the $\mathrm{Y}^{3+} / \mathrm{Eu}^{3+}$ coordination polyhedra leading to high contribution of the electric dipole transition. A higher niobium content host lattice is associated with a decrease of the oxygen vacancy rate within the matrix. Thus, the local environment of the doping element is supposed

related to a lowest crystallinity of the materials, but it can be attributed to the heating mode (natural heating in a standard furnace) that does not exacerbate ion mobility like in SPS sintering. Conversely, the specificity of the SPS technique results in the stabilization of two fluorite end- to become less defective and thus more regular for the highest niobium content. A higher $\mathrm{M}-\mathrm{O}$ bond covalency leads to stabilize the $\mathrm{O}(2 \mathrm{p})$ band.

In parallel a more regular coordination polyhedron of the europium ions results in a decrease of the chemical bonding average length between europium and oxygen ions. The europium element recovers a central position within its coordination polyhedron. An increased covalent character of this chemical bonding stabilizes the $4 \mathrm{f}$ orbitals, which tends to reduce the $5 d-4 f$ orbital mixing. This facilitates the $\mathrm{Eu}^{3+}-\mathrm{O}$ CTB, which shifts to the lower energy as observed in Figure $7 \mathrm{~b}$. In addition, this impacts the electric dipole transition probabilities. By consequence, even if the main part of the emission is still due to the electric dipole transition, the ratio between the magnetic dipole transition and the previous ones is expected to be higher for the highest niobium content matrix (Figure 7). The highest niobium content phase should also present the lowest ionic conductivity as reported in ref 10 .

Excitations in the $4 \mathrm{f}$ lines reveal for both compositions the presence of impurity contribution. The lowest niobium content emission graph (Figure $7 b$ ) shows lines of $\mathrm{Eu}^{3+}$-doped $\mathrm{Y}_{2} \mathrm{O}_{3}$ sesquioxide, whereas the $27 \%$ compound preparation contains $\mathrm{Eu}^{3+}$-doped $\mathrm{YNbO}_{4}$ (Figure 7c) in agreement with XRD analyses and with the known $\mathrm{Y}_{2} \mathrm{O}_{3}-\mathrm{Nb}_{2} \mathrm{O}_{5}$ phase diagram. This tentative phase diagram actually shows a $\mathrm{Y}_{2} \mathrm{O}_{3}-$ $\mathrm{Y}_{3} \mathrm{NbO}_{7}$ biphasic domain for $\mathrm{Nb}$ compositions lower than $\sim 20 \%$ and $\mathrm{Y}_{3} \mathrm{NbO}_{7}-\mathrm{YNbO}_{4}$ biphasic domain for $\mathrm{Nb}$ compositions higher than $\sim 31 \%$.(9) As said previously, these secondary phases result from the partial phase decomposition of the $\mathrm{Y}_{3} \mathrm{NbO}_{7}$ intermediate phase at $1600{ }^{\circ} \mathrm{C}$.(8) But whatever the excitation wavelengths in the CTB or the $4 f$ absorption lines, for one given composition, the emission curves are perfectly superimposed and do not present coexistence between the 1 and 2 emissions as observed previously on the SPS sintered pellet (Figure 7c,d). This is confirmed by the XRD patterns of the powders (Figure S4), which do not present splitting of the diffraction peaks at the highest angles. This is not

compositions of the niobate intermediate phase. We can conclude that the composition splitting into a low and a high niobium content fluorite-type composition is induced or accelerated by the SPS process and the post-reoxidation process. The SPS experiments seem 
to reveal the existence of a lack of miscibility at 1600

${ }^{\circ} \mathrm{C}$ between $24 \%$ and a $28-29 \%$ of niobium content.

Interdiffusions of different phases within complex architecture pellets as sandwich pellets prepared by SPS and reactive sintering were observed previously. The corresponding studies clearly highlight the correlation between the direction of diffusion, creation of interphases, depletion zone, and the current sense or current pulses frequency. $(25,26)$ Moreover in these studies, intermediate phases are stabilized in the interphase because of a reactive sintering. In the present investigation, the experimental conditions make possible the preservation of the niobate solid solution but create a composite system leading to tune the luminescent properties.

\section{Conclusion}

The occurrence of two spectral fingerprints of europium emissions was enhanced on a pellet sintered through SPS process. This is the consequence of a phase splitting into two fluorite-type compositions in which environments of the rare earth ions slightly differ due to the different niobium content. The intrinsic disorder due to the occurrence of oxygen vacancies results in amorphous-type responses of the luminescent cations, and it varies as a function of niobium content. These observations are also supported by electron diffraction patterns (TEM) that show grain inhomogeneity within the observed materials. Moreover XRD diagram refinements show the occurrence of two fluorite phases where the cell parameters can be directly related to the niobium concentration. The local cubic environment of europium ions appears noncentrosymmetric due to the oxygen vacancies.

Because such an optical response is not observed in powders heated under the same conditions of time and temperature in a standard furnace, we can conclude that this is related to the SPS sintering process, pressure coupled with a current effect, which activates or accelerates oxygen mobility and cation diffusion resulting into the stabilization of two close compositions of the initial niobate phase. These analyses seem to confirm the existence of a lack of miscibility at high temperature in the phase diagram. Finally the lowest niobium rate (24\%) stabilized in this fluorite-type phase corresponds also to the highest conductivity in the $\mathrm{Y} 2 \mathrm{O} 3-\mathrm{Nb} 2 \mathrm{O} 5$ systems on the basis of the literature.

\section{Funding Information}

The authors thank the CNRS, the Aquitaine region, and the pôle de compétitivité Routes de lasers for their support. This study was performed with financial support from the French State, managed by the French National Research Agency (ANR) in the frame of "the Investments for the future" Program IdEX Bordeaux-LAPHIA (ANR-10-IDEX-03-02), EXOLAS project.

\section{Acknowledgements}

The authors thank E. Lebraud, ICMCB-CNRS, for the Xray diffraction pattern acquisition and $A$. Fargues, $I C M C B$, for luminescence setup calibration section.

\section{References}

1. Rossell, H. J.Fluorite-related phases $\mathrm{Ln}_{3} \mathrm{MO}_{7}, \mathrm{Ln}=$ rare earth, Y or Sc, M = Nb, Sb, or Ta: II. Structure determination J. Solid State Chem. 1979, 27, 115- 122, DOI: 10.1016/0022-4596(79)90150-6

2. Doi, Y.; Harada, Y.; Hinatsu, Y.Crystal structures and magnetic properties of fluorite-related oxides $\mathrm{Ln}_{3} \mathrm{NbO}_{7}$ (Ln = lanthanides) J. Solid State Chem. 2009, 182, 709715, DOI: 10.1016/j.jssc.2008.12.012

3. López-Conesa, L.; Rebled, J. M.; Chambrier, M. H.; Boulahya, K.; González-Calbet, J. M.; Braida, M. D.; Dezanneau, G.; Estradé, S.; Peiró, F.Local Structure of Rare Earth Niobates $\left(\mathrm{RE}_{3} \mathrm{NbO}_{7}, \mathrm{RE}=\mathrm{Y}, \mathrm{Er}, \mathrm{Yb}, \mathrm{Lu}\right)$ for Proton Conduction Applications Fuel Cells 2013, 13, 29-33, DOI: 10.1002/fuce.201200136

4. Miida, R.; Sato, F.; Tanaka, M.; Naito, H.; Arashi, H.Locally Modulated Structures of Fluorite-Related $\mathrm{Y}_{2} \mathrm{O}_{3}-\mathrm{Nb}_{2} \mathrm{O}_{5}$ Solid Solutions J. Appl. Crystallogr. 1997, 30, 272- 279, DOI: 10.1107/S0021889896013283

5. Allpress, J. G.; Rossell, H. J.Fluorite-related phases $\mathrm{Ln}_{3} \mathrm{MO}_{7}, \mathrm{Ln}=$ rare earth, $\mathrm{Y}$, or Sc, $\mathrm{M}=\mathrm{Nb}, \mathrm{Sb}$, or Ta. I. Crystal chemistry J. Solid State Chem. 1979, 27, 105114, DOI: 10.1016/0022-4596(79)90149-X

6. Withers, R. L.; Thompson, J. G.; Barlow, P. J.; Barry, J. C.The 'Defect Fluorite' phase in the $\mathrm{ZrO}_{2}-\mathrm{PrO}_{1.5}$ system and its relationship to the structure of pyrochlore Aust. J. Chem. 1992, 45, 1375-1395, DOI: 10.1071/CH9921375

7. Kovyazina, S. A.; Perelyaeva, L. A.; Leonidov, I. A.; Bakhteeva, Y. A.High-Temperature Structural Disorder in $\mathrm{R}_{3} \mathrm{NbO}_{7} \mathrm{~J}$. Struct. Chem. 2003, 44, 975-979, DOI: 10.1023/B:JORY.0000034803.89136.ba

8. Kim, K.-Y.; Durand, A.; Heintz, J.-M.; Veillere, A.; Jubera, V.Spectral evolution of $\mathrm{Eu}^{3+}$ doped $\mathrm{Y}_{3} \mathrm{NbO}_{7}$ niobate induced by temperature J. Solid State Chem. 2016, 235, 169- 174, DOI: 10.1016/j.jssc.2015.12.023

9. Babich, T. G.; Zagorodnyuk, A. V.; Teterin, G. A.; Sadkovskaya, L. V.; Zhirnova, A. P.The $\mathrm{Y}_{2} \mathrm{O}_{3}-\mathrm{Nb}_{2} \mathrm{O}_{5}$ system Ukr. Khim. Zh. 1989, 55, 132-134. (translated Sov. Prog. Chem. 1989, 55(2), 21-24)

10. Lee, J.-H.; Yashima, M.; Kakihana, M.; Yoshimura, M.Phase Diagram and Oxygen-Ion Conductivity in the $\mathrm{Y}_{2} \mathrm{O}_{3}-\mathrm{Nb}_{2} \mathrm{O}_{5}$ System J. Am. Ceram. Soc. 1998, 81, 894900, DOI: 10.1111/j.1151-2916.1998.tb02424.x 
11. Rodriguez-Carvajal, J. Commission on Powder Diffraction (IUCr) Newsletter; IUCr, 2001.

12. Jubera, V.; Chaminade, J. P.; Garcia, A.; Guillen, F.; Fouassier, C.Luminescent properties of Eu3+-activated lithium rare earth borates and oxyborates J. Lumin. 2003, 101, 1- 10, DOI: 10.1016/S0022-2313(02)003356

13. Hirano, M.; Dozono, H.Direct Formation and Luminescence Properties of Yttrium Niobate $\mathrm{YNbO}_{4}$ Nanocrystals via Hydrothermal Method (pages 33893393) J. Am. Ceram. Soc. 2013, 96, 3389-3393, DOI: 10.1111/jace.12595

14. Mc Cauley, R. A.; Hummel, F. A.Luminescence as an indication of distortion in $\mathrm{A}^{3+}{ }_{2} \mathrm{~B}^{4+}{ }_{2} \mathrm{O}_{7}$ type pyrochlores J. Lumin. 1973, 6, 105- 115, DOI: 10.1016/00222313(73)90046-X

15. Hirayama, M.; Sonoyama, N.; Yamada, A.; Kanno, R. Relationship between structural characteristics and photoluminescent properties of $\left(\mathrm{La}_{1-\mathrm{x}} \mathrm{Eu}_{\mathrm{x}}\right)_{2} \mathrm{M}_{2} \mathrm{O}_{7}(\mathrm{M}=$ $\mathrm{Zr}, \mathrm{Hf}, \mathrm{Sn})$ pyrochlores J. Lumin. 2008, 128, 18191825, DOI: 10.1016/j.jlumin.2008.05.005

16. Fornasiero, P.; Speghini, A.; Di Monte, R.; Bettinelli, M.; Kašpar, J.; Bigotto, A.; Sergo, V.; Graziani, M.LaserExcited Luminescence of Trivalent Lanthanide Impurities and Local Structure in $\mathrm{CeO}_{2}-\mathrm{ZrO}_{2}$ Mixed Oxides Chem. Mater. 2004, 16, 1938-1944, DOI: 10.1021/cm035370b

17. Montini, T.; Speghini, A.; De Rogatis, L.; Lorenzut, B.; Bettinelli, M.; Graziani, M.; Fornasiero, P.Identification of the Structural Phases of $\mathrm{Ce}_{x} \mathrm{Zr}_{1-\mathrm{x}} \mathrm{O}_{2}$ by Eu(III) Luminescence Studies J. Am. Chem. Soc. 2009, 131, 13155-13160, DOI: 10.1021/ja905158p

18. Blasse, G.On the luminescence of bis (triphenylphosphine) phenanthroline copper (I) Chem. Phys. Lett. 1980, 70, 1-3, DOI: 10.1016/00092614(80)80047-9

19. Koepke, C.; Wojtowicz, A. J.; Lempicki, J.Excited-state absorption in excimer-pumped $\mathrm{CaWO}_{4}$ crystals J. Lumin. 1993, 54, 345- 355, DOI: 10.1016/00222313(93)90003-6

20. Glorieux, B.; Jubera, V.; Apheceixborde, A.; Garcia, A.Luminescence properties of tungstates and molybdates phosphors: Illustration on $\mathrm{ALn}\left(\mathrm{MO}_{4}\right)_{2}$ compounds $(A=$ alikaline cation, $\mathrm{Ln}=$ lanthanides, $\mathrm{M}=$ W, Mo) Solid State Sci. 2011, 13, 460-467, DOI: 10.1016/j.solidstatesciences.2010.12.013

21. Barker, W. W. The systems $\mathrm{Y}_{2} \mathrm{O}_{3}-\mathrm{Nb}_{2} \mathrm{O}_{5}$ and $\mathrm{Ho}_{2} \mathrm{O}_{3}-$ $\mathrm{Nb}_{2} \mathrm{O}_{5}$ : the cubic solid-solution region J. Mater. Sci. Lett. 1984, 3, 492-494, DOI: 10.1007/BF00720980

22. García-Martin, S.; Alario-Franco, M. A.; Fagg, D. P.; Feighery, A. J.; Irvine, J. T. S.Modulated Fluorite-Type Structure of Materials from the $(1-\mathrm{x}) \mathrm{Y}_{0.5} \mathrm{Zr}_{0.5} \mathrm{O}_{1.75}$ $\mathrm{xY}_{0.75} \mathrm{Nb}_{0.25} \mathrm{O}_{1.75}(0 \leq x \leq 1)$ System Chem. Mater. 2000 12, 1729- 1737, DOI: $10.1021 / \mathrm{cm} 991204$

23. Withers, R. L.; Thompson, J. G.; Barlow, P. J.An electron, and $\mathrm{X}$-ray powder, diffraction study of cubic, fluorite-related phases in various $\mathrm{ZrO}_{2}-\mathrm{Ln}_{2} \mathrm{O}_{3}$ systems $\mathrm{J}$. Solid State Chem. 1991, 94, 89-105, DOI: 10.1016/0022-4596(91)90224-6

24. Cai, L.; Nino, J. C.Structure and dielectric properties of $\mathrm{Ln}_{3} \mathrm{NbO}_{7}(\mathrm{Ln}=\mathrm{Nd}, \mathrm{Gd}, \mathrm{Dy}, \mathrm{Er}, \mathrm{Yb}$ and $\mathrm{Y})$ J. Eur. Ceram. Soc. 2007, 27, 3971-3976, DOI: 10.1016/j.jeurceramsoc.2007.02.077

25. Santanach, J. G.; Estournès, C.; Weibel, A.; Chevallier, G.; Bley, V.; Laurent, C.; Peigney, A.Influence of pulse current during Spark Plasma Sintering evidenced on reactive alumina-hematite powders J. Eur. Ceram. Soc. 2011, 31, 2247-2254, DOI: 10.1016/j.jeurceramsoc.2011.06.003

26. Albino, M.; Chung, U.-C.; Kassas, A.; Lesseur, J.; Bernard, D.; Elissalde, C.; Maglione, M.You have full text access to this content Electric Current as a Driving Force for Interphase Growth in Spark Plasma Sintered Dielectric Composites J. Am. Ceram. Soc. 2016, 99, 406- 409, DOI: 10.1111/jace.14052

\section{Supporting Information}

The Supporting Information is available free of charge on the ACS Publications website at DOI: 10.1021/acs.inorgchem.7b00088. 


\section{Table 1. $\mathrm{Eu}^{3+}$ Transitions Observed at $4 \mathrm{~K} / 300 \mathrm{~K}$}

\begin{tabular}{|c|c|c|}
\hline transitions & emission $1 \lambda_{\text {exc }}=462 \mathrm{~nm}$ & emission $2 \lambda_{\mathrm{exc}}=468 \mathrm{~nm}$ \\
\hline${ }^{5} \mathrm{D}_{2}-{ }^{7} \mathrm{~F}_{2}$ & $495-503$ & $495-503$ \\
\hline${ }^{5} \mathrm{D}_{2}-{ }^{7} \mathrm{~F}_{3}$ & $506-522$ & $506-522$ \\
\hline${ }^{5} \mathrm{D}_{1}-{ }^{7} \mathrm{~F}_{1}$ & $522-546$ & $522-546$ \\
\hline${ }^{5} \mathrm{D}_{1}-{ }^{7} \mathrm{~F}_{2}$ & $546-560$ & $546-560$ \\
\hline${ }^{5} \mathrm{D}_{1}-{ }^{7} \mathrm{~F}_{3}$ & 568 & 568 \\
\hline${ }^{5} \mathrm{D}_{0}-{ }^{7} \mathrm{~F}_{0}$ & $576^{a}$ & $580^{a}$ \\
\hline \multirow[t]{3}{*}{${ }^{5} \mathrm{D}_{0}-{ }^{7} \mathrm{~F}_{1}$} & 582 & 587 \\
\hline & 589.2 & 592 \\
\hline & & 598 \\
\hline \multirow[t]{3}{*}{${ }^{5} \mathrm{D}_{0}-{ }^{7} \mathrm{~F}_{2}$} & 600 & 610 \\
\hline & 605.4 & 629.2 \\
\hline & 629.4 & \\
\hline \multirow[t]{2}{*}{${ }^{5} \mathrm{D}_{0}-{ }^{7} \mathrm{~F}_{3}$} & 642.7 & 655 \\
\hline & 656.6 & \\
\hline \multirow[t]{4}{*}{${ }^{5} \mathrm{D}_{0}-{ }^{7} \mathrm{~F}_{4}$} & 682 & 682 \\
\hline & 695.8 & 696.4 \\
\hline & 707.6 & 702.8 \\
\hline & 712.8 & 711.2 \\
\hline
\end{tabular}

${ }^{a}$ Uncertainty remains in the attribution of these lines because of the presence of a thermal population of the ${ }^{7} \mathrm{~F}_{1}$ at room temperature.

Table 2. Data Resulting from the Rietveld Refinements ${ }^{a}$ on Fluorite Phases

\begin{tabular}{lcc} 
& \multicolumn{1}{c}{ fluorite 1} & fluorite 2 \\
cell parameters & $a=5.2525(9) \AA$ & $a=5.2398(2) \AA$ \\
Bragg R factor & 8.47 & 8.12 \\
FR factor & 6.23 & 6.15 \\
\multicolumn{2}{c}{ conventional Rietveld R-factor $R p=15.4, R w p=15.1, R_{\text {exp }}=7.5$} \\
relative fluorites' proportion & $77.9 \%$ & $22.1 \%$
\end{tabular}

${ }^{a}$ Refinements were performed by fixing the atomic position at the general 000 and $1 / 41 / 41 / 4$ positions for the fluorite matrix. 

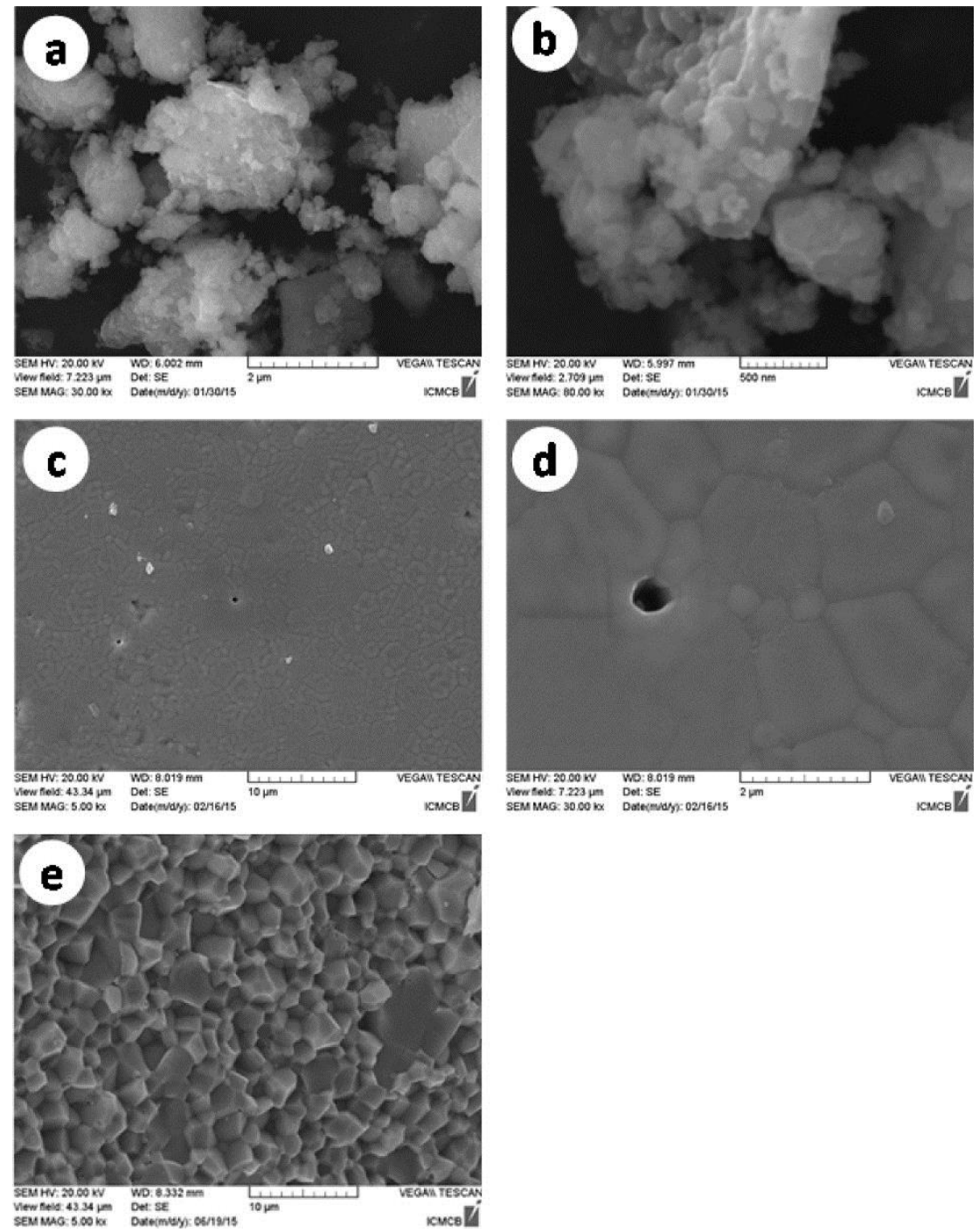

Figure 1. SEM pictures of initial powder $(a, b)$, surface $(c, d)$, and fracture $(e)$ of the $\mathrm{Eu}^{3+}$-doped niobate. The sintering of nanometric grains results in the obtaining of dense ceramic. 

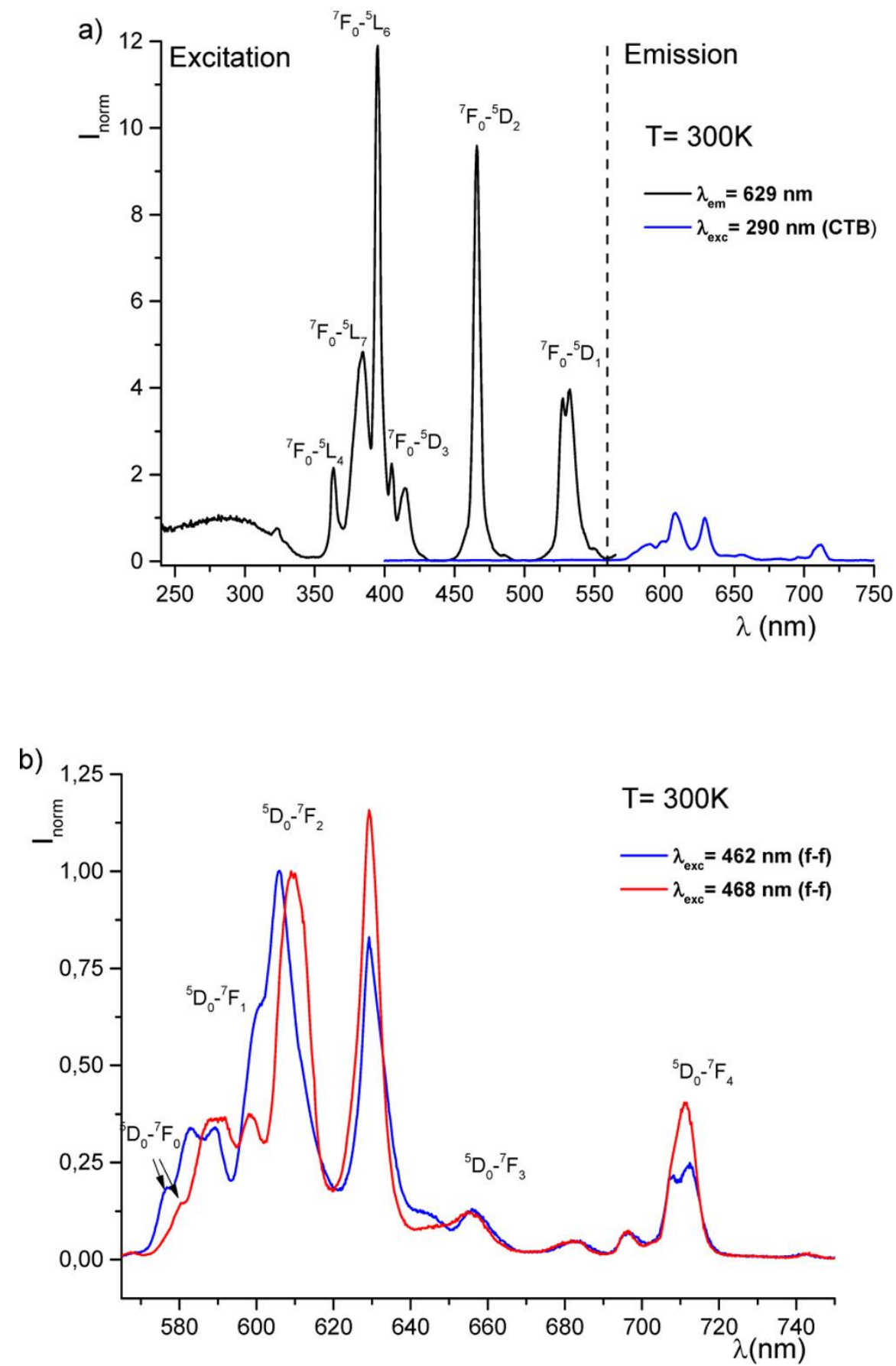

Figure 2. Luminescence spectra of Eu $u^{3+}$-doped $\mathrm{Y}_{3} \mathrm{NbO}_{7}$ pellet. Excitation graph (a, left) shows a strong quenching of the charge transfer band (СТВ). Excitations in the CTB (a, right) or in the $4 \mathrm{f} \mathrm{lines} \mathrm{(b)} \mathrm{lead} \mathrm{to} \mathrm{unstructured} \mathrm{emission}$ lines maximum in the red range. 
a)

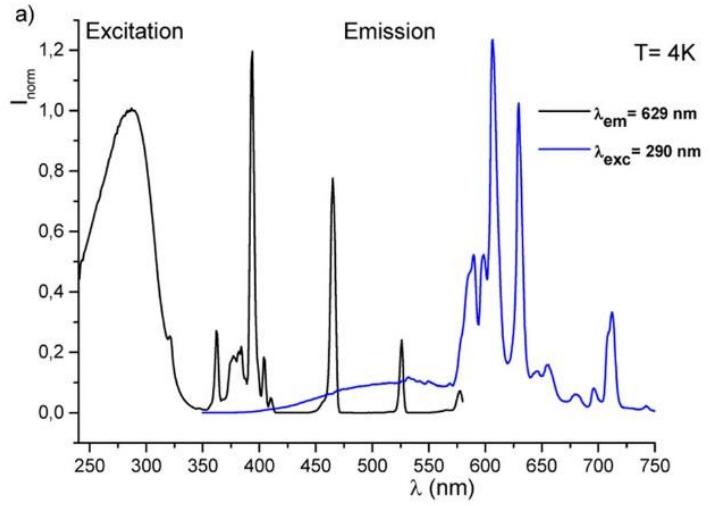

c)

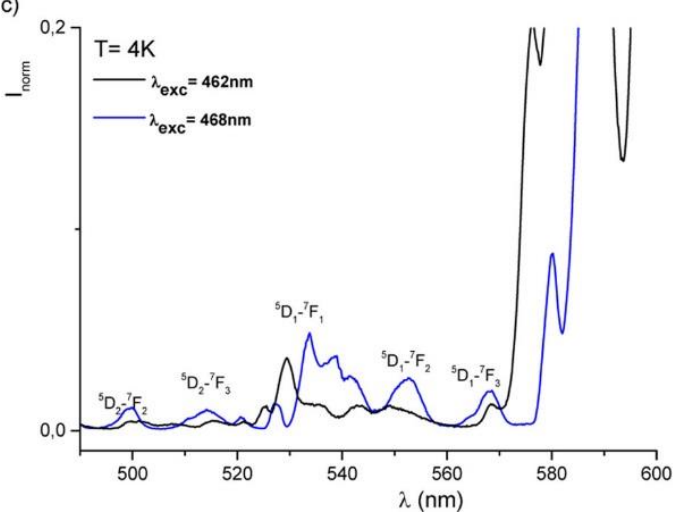

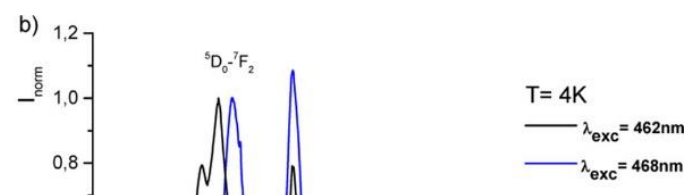

${ }^{5} \mathrm{D}_{0}{ }^{7} \mathrm{~F}_{4}$

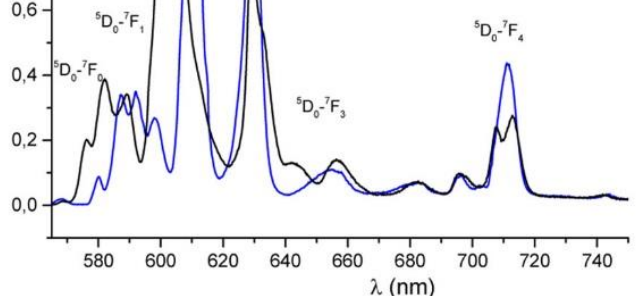

d)

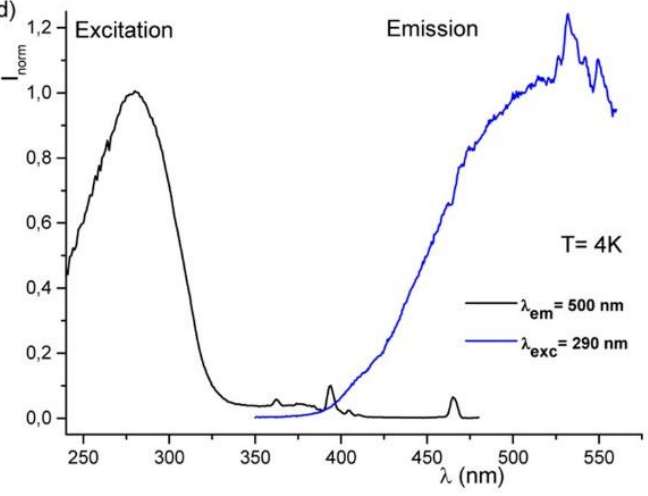

Figure 3. Luminescence spectra of $\mathrm{Eu}^{3+}$-doped $\mathrm{Y}_{3} \mathrm{NbO}_{7}$ pellet at $4 \mathrm{~K}$. (a-d) Excitation and emission graphs performed at the maximum intensities (a), emission graphs corresponding to excitation in the $4 \mathrm{f}$ lines of $\mathrm{Eu}^{3+}$ (b) and enlargement (c). The low-temperature measurements put in evidence the configurational quenching of the emission (a) and an additional matrix contribution (d). 
a)
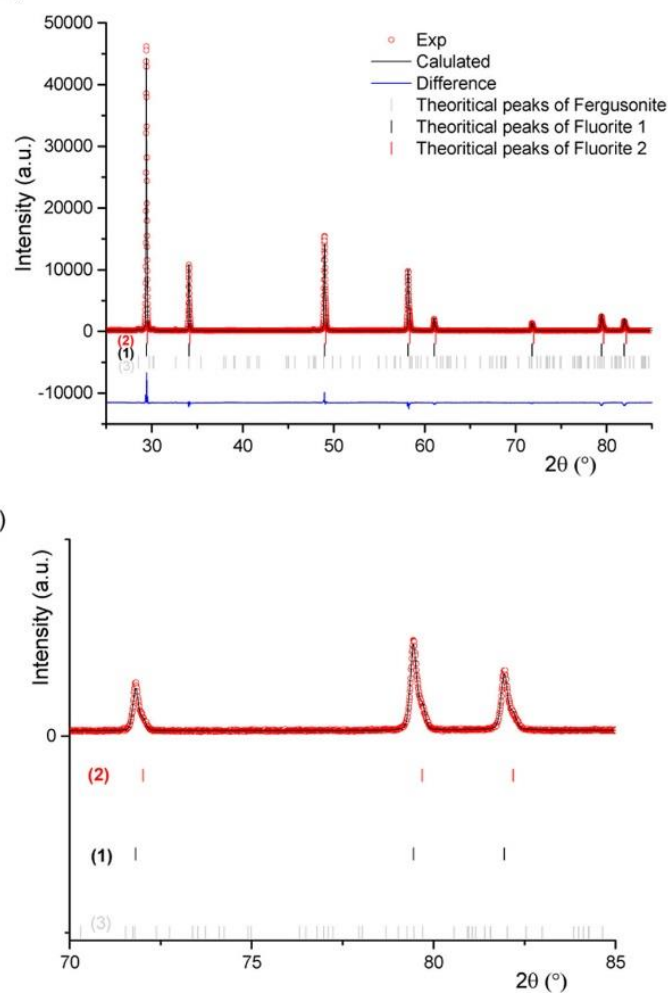

b)

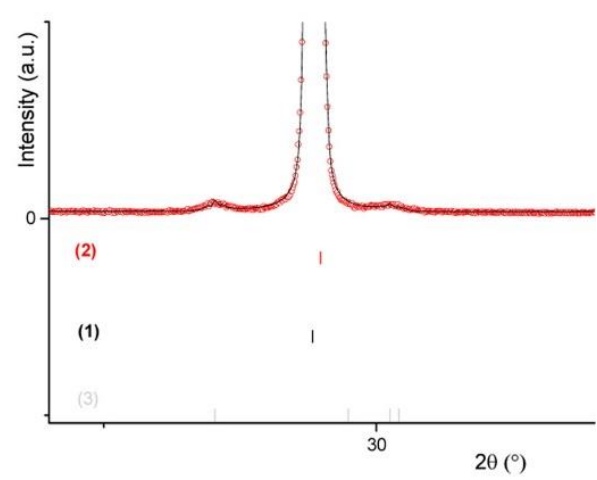

Figure 4. (a) Rietveld refinement of $\mathrm{E} \mathrm{Eu}^{3+}(5 \mathrm{~mol} \%)$-doped $\mathrm{Y}_{3} \mathrm{NbO}_{7}$ pellet sintered at $1600{ }^{\circ} \mathrm{C}$. (b, c) Enlargement of the lowest and highest angle regions, respectively. A splitting of the diffraction peaks is visible in the high-angle region.

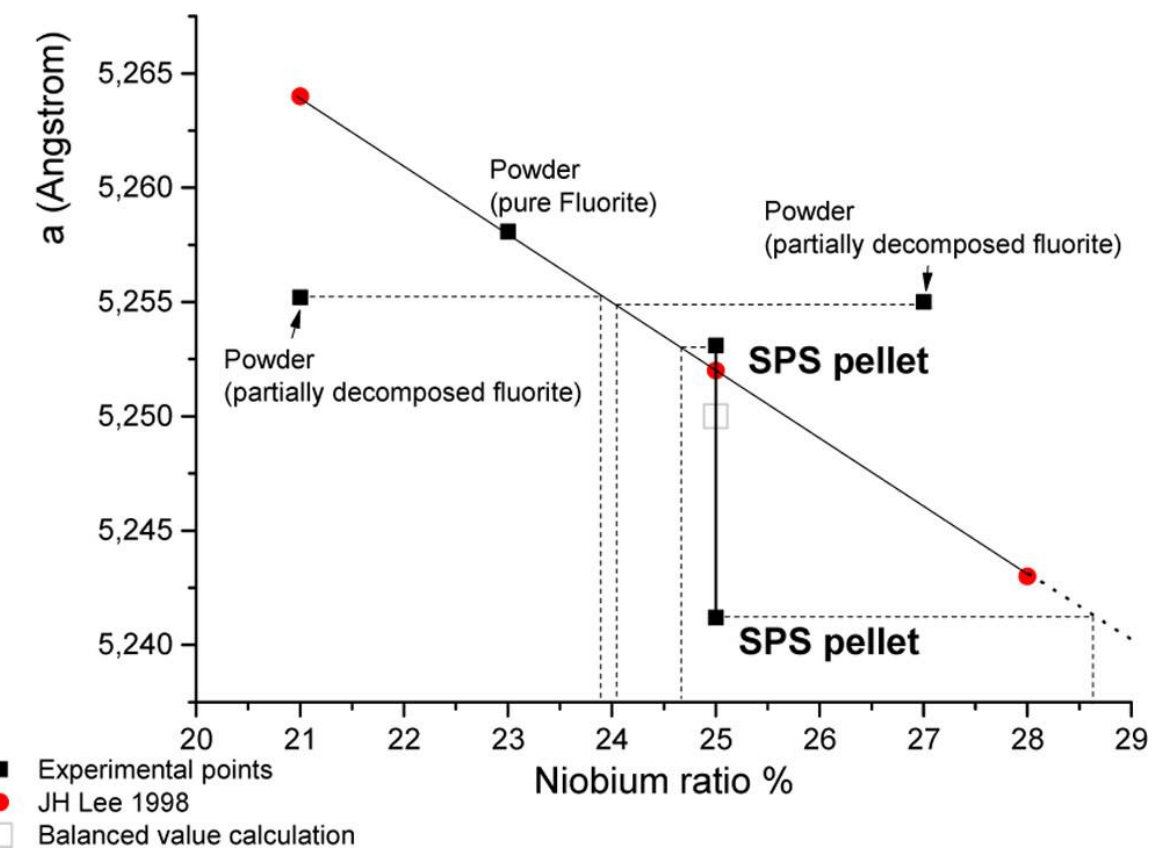

Figure 5. Evolution of the $a$ cell parameter as a function of niobium content in niobate compounds. The vertical full line corresponds to the splitting of the diffraction peaks observed for SPS sintered samples. Linear variation is derived from the data of J.-H. Lee.(10) 


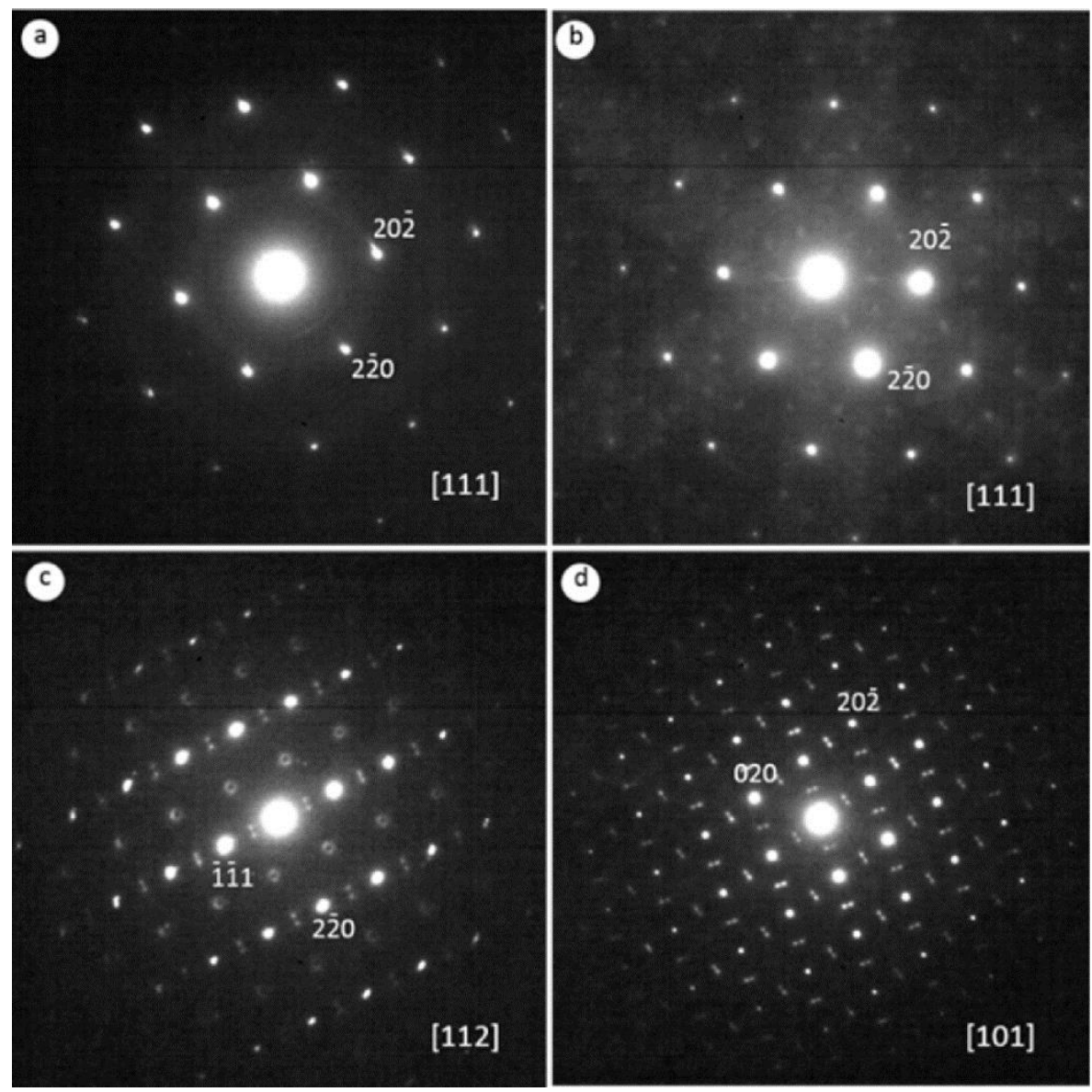

Figure 6. Electron diffraction patterns of the Eu ${ }^{3+}$-doped $\mathrm{Y}_{3} \mathrm{NbO}_{7}$ pellet: Bragg reflections observed in the [111] direction consistent with the fluorite Fm3m cell (a) and additional diffuse and localized spots in the same orientation (b), patterns observed in the [112] (c) and [101] (d) directions. Inhomogeneity of the grains is evidenced. 

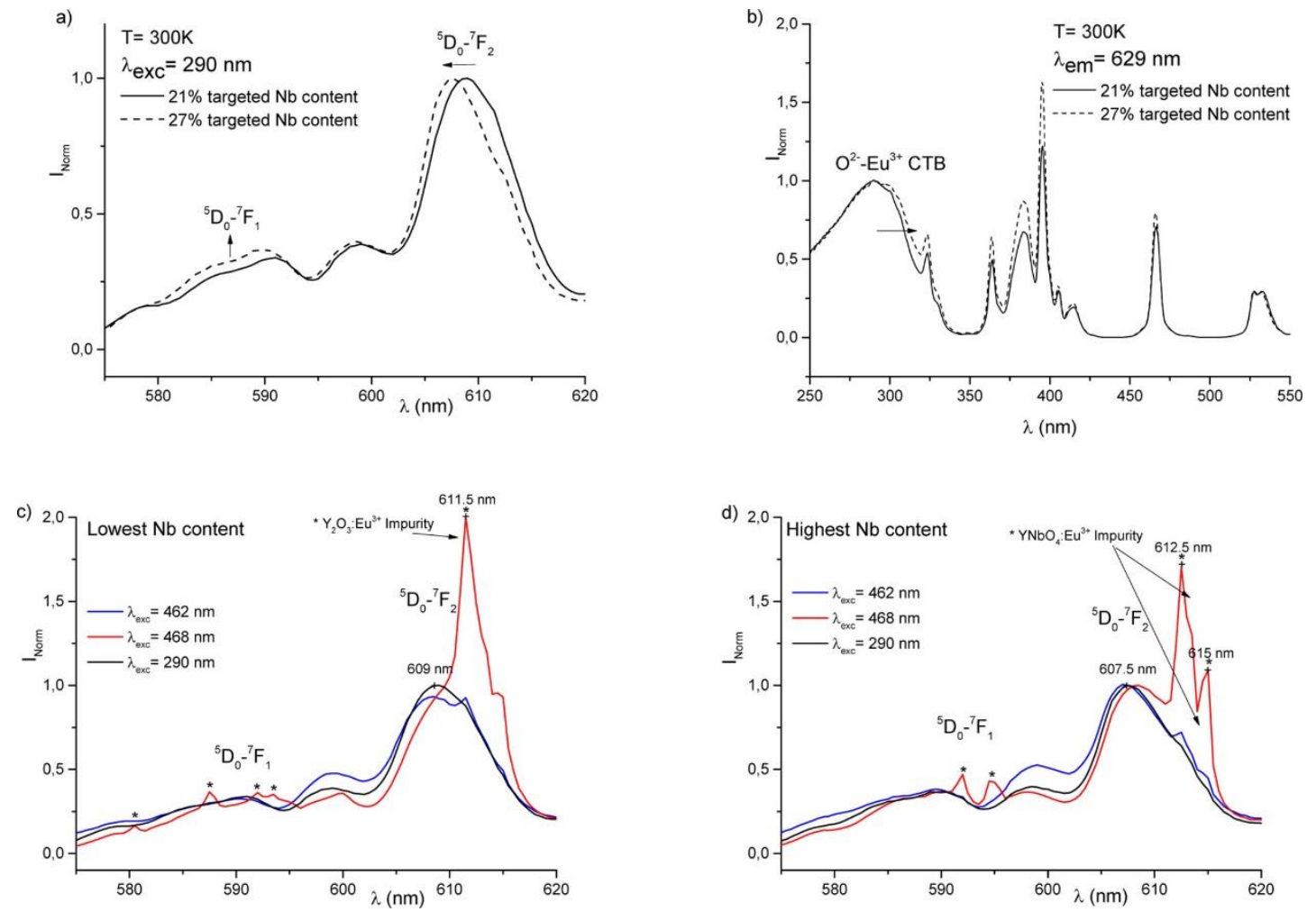

Figure 7. Room-temperature emission and excitation of $\mathrm{Eu}^{3+}$ 5\%-doped $\mathrm{Y}_{3} \mathrm{Nb}_{0.8} \mathrm{O}_{6.5}$ (21\% of niobium) and $\mathrm{Y}_{3} \mathrm{Nb}_{1.125} \mathrm{O}_{7.5}$ (27\% of niobium) powders under excitation in the CTB at $290 \mathrm{~nm}$ (a) and emission at the maximum (b), respectively. Superimposed normalized emission curves of both compositions for excitation in the CTB and the $4 \mathrm{f}$ absorption lines of $\mathrm{Eu}^{3+}(\mathrm{b}, \mathrm{c})$. A unique response related to the niobate solid solution is detected in addition to trace of impurities due to partial decomposition. 